\title{
Lycopene overproduction and in situ extraction in organic-aqueous culture systems using a metabolically engineered Escherichia coli
}

Julia Gallego-Jara ${ }^{\dagger}$,Teresa de Diego*† ${ }^{*}$ Álvaro del Real, Ana Écija-Conesa, Arturo Manjón and Manuel Cánovas

\begin{abstract}
Lycopene is an important compound with an increasing industrial value. However, there is still no biotechnological process to obtain it. In this study, a semi-continuous system for lycopene extraction from recombinant Escherichia coli BL21 cells is proposed. A two-phase culture mode using organic solvents was found to maximize lycopene production through in situ extraction from cells. Within the reactor, three phases were formed during the process: an aqueous phase containing the recombinant $E$. coli, an interphase, and an organic phase. Lycopene was extracted from the cells to both the interphase and the organic phase and, consequently, thus enhancing its production. Maximum lycopene production $\left(74.71 \pm 3.74 \mathrm{mg} \mathrm{L}^{-1}\right)$ was obtained for an octane-aqueous culture system using the $E$. coli BL21LF strain, a process that doubled the level obtained in the control aqueous culture. Study of the interphase by transmission electron microscopy (TEM) showed the proteo-lipidic nature and the high storage capacity of lycopene. Moreover, a cell viability test by flow cytometry (CF) after $24 \mathrm{~h}$ of culture indicated that $24 \%$ of the population could be re-used. Therefore, a batch series reactor was designed for semi-continuous lycopene extraction. After five cycles of operation (120 h), lycopene production was similar to that obtained in the control aqueous medium. A final specific lycopene yield of up to $49.70 \pm 2.48 \mathrm{mg} \mathrm{g}^{-1}$ was reached at $24 \mathrm{~h}$, which represents to the highest titer to date. In conclusion, the aqueous-organic semi-continuous culture system proposed is the first designed for lycopene extraction, representing an important breakthrough in the development of a competitive biotechnological process for lycopene production and extraction.
\end{abstract}

Keywords: Lycopene, Metabolically engineered Escherichia coli, Fed-batch culture, Organic-aqueous culture system

\section{Introduction}

Lycopene is a tetraterpenoid (C40) precursor of carotenoids. Traditionally, it was considered a colorant and a food additive, but new applications have been proposed for use as an antioxidant (Chasse et al. 2001) and anticarcinogen (Giovannucci et al. 2002; Rabi and Gupta 2008) and for preventing against cardiovascular diseases (Rao 2002), hepatic fibro-genesis (Kitade et al. 2002) or human

\footnotetext{
*Correspondence: tdp@um.es

${ }^{\dagger} J$ ulia Gallego-Jara and Teresa de Diego contributed equally to this work Department of Biochemistry and Molecular Biology (B) and Immunology, Faculty of Chemistry, University of Murcia, Campus de Espinardo, Regional Campus of International Excellence "Campus Mare Nostrum", P.O. Box 4021, 30100 Murcia, Spain
}

papillomavirus persistence (Sedjo et al. 2002). In spite of its great importance, most of lycopene is obtained from tomato, and no a competitive biotechnological exists process for its production.

In industry, methods based on metabolic engineering are the most profitable due to their high productivity and, consequently, the search for a biotechnological method for lycopene production is an important challenge for many researchers. In the last decade, many studies have been reported concerning lycopene production by metabolic engineering, some of which are based on E. coli, the most important cell factory microorganism in biotechnology (Kim and Keasling 2001; Martin et al. 2003; Alper et al. 2006; Yuan et al. 2006;

\section{照 Springer}


Rodriguez-Villalon et al. 2008; Yoon et al. 2008; Zhou et al. 2012; Chen et al. 2013). Recombinant E. coli are capable of biosynthesizing lycopene through either the mevalonate (MEP) or the non-mevalonate route. Although the $E$. coli possesses the genes of the nonmevalonate route (or 2-C-methyl-D-erythritol 4-phosphate pathway) for isopentenyl pyrophosphate (IPP) synthesis, is still requires the following three enzymes, geranylgeranyl pyrophosphate (GGPP) synthase (crtE), phytoene synthase $(c r t \mathrm{~B})$ and phytoene desaturase (crtI) to be able to synthesize lycopene. Therefore, a recombinant bacterium which contains these enzymes is necessary (Additional file 1: Figure S1). Previous works demonstrated that carotenoid production can be improved by increasing the amount of IPP and its isomer dimethylallyl pyrophosphate (DMAPP) amount available in the recombinant E. coli engineered (Jin and Stephanopoulos 2007; Zhou et al. 2012; Zhang et al. 2013). Besides to E. coli, carotenogenic microorganisms, such as Blakeslea trispona (Xu et al. 2007) and the non-carotenogenic yeasts, Pichia pastoris (Araya-Garay et al. 2012) and Saccharomyces cerevesiae (Bahieldin et al. 2014), have been used to produce lycopene.

Despite the achievements made to date, there is still no competitive biotechnological method to compete with lycopene extraction from tomatoes. The main problems of these metabolic engineering processes are plasmid instability and the low capability to accumulate lycopene in the cytoplasmic membrane from non-carotenogenic organisms (Wang et al. 2012). Hence, the in situ recovery of lycopene from a recombinant $E$. coli strain is the goal for achieving a competitive biotechnological process. To our knowledge there are few studies concerning lycopene extraction from $E$. coli. It has been reported an in situ process based on lycopene overproduction and recovery using octane and decane as extraction solvents (Yoon et al. 2008). However, the lycopene percentage extracted was quite low. The partial digestion of bacterial walls with lysozyme improved the system extractive capacity, although this digestion was quickly reverted as bacteria duplicated. In 2011, an in situ extraction process of retinoids from E. coli was reported (Jang et al. 2011), in which dodecane was used as extraction solvent, attaining a 68 -fold higher productivity than attained with the aqueous system.

Hence, the search for a competitive system concerning both lycopene production and extraction is of great interest in the biotechnology field. In this paper, we propose the first semi-continuous system to produce and extract high amounts of lycopene employing a recombinant $E$. coli strain.

\section{Materials and methods}

\section{Cell mass and specific growth rate}

Cell mass was determined using a linear calibration curve relating optical density at $600 \mathrm{~nm}$ (OD600) and dry cell weight $\left(R^{2}=0.99\right)$. Cells were filtered and washed thoroughly with distilled water, and then dried at $130{ }^{\circ} \mathrm{C}$ for $24 \mathrm{~h}$ to a constant weight using a thermobalance (Electronic Moisture Analyzer model MA35, Sartorius). The exponential growth phase was identified and the specific growth rate was determined for all culture strains cultures (Sauer et al. 1999).

\section{Transformation and culture conditions}

Chemically competent E. coli K12 (BW25113) (Baba et al. 2006) and BL21-Gold (DE3) (Agilent Technologies) cells were transformed with the pAC-Lyc plasmid, which contained three genes of the lycopene pathway, $\operatorname{crtE}, \operatorname{crt} B$ and $c r t I$, and a chloramphenicol resistance gene, by heat shock at $42{ }^{\circ} \mathrm{C}$. The resulting strains were called E. coli K12L and E. coli BL21L, respectively. Then, E. coli BL21L was made competent again and co-transformed with the pET-SIDF and pET-SIDFG plasmids, obtaining the strains E. coli BL21LF and E. coli BL21LG, respectively (Table 1). These plasmids contained the genes $d x s$, idi, ispD, ispF (pET-SIDF) and $d x s$, idi, ispD, ispF and isp G (pET-SIDFG) and an expression plasmid controlled by the inducible promoter T7. Besides, they showed ampicillin resistance. The plasmids pAC-Lyc, pET-SIDF and pET-SIDFG were kindly supplied by Prof. G. Stephanopoulos (Department of Chemical Engineering, Institute of Technology, Cambridge, Massachusetts, EEUU) (Zhou et al. 2012).

Lycopene biosynthesis was carried out in triplicate in $500 \mathrm{~mL}$ flasks containing $50 \mathrm{~mL}$ of MM9 medium with $20 \mathrm{mM}$ glucose or $40 \mathrm{mM}$ glycerol using an orbital shaking at $200 \mathrm{rpm}$ and $28^{\circ} \mathrm{C}$. The culture medium was supplemented with appropriate antibiotics $\left(30 \mu \mathrm{g} \mathrm{mL} \mathrm{m}^{-1}\right.$ chloramphenicol and/or $100 \mu \mathrm{g} \mathrm{mL}^{-1}$ ampicillin).

\section{Lycopene extraction}

Metabolically engineered $E$. coli cells were harvested by centrifugation at $10,000 \times g$ for $5 \mathrm{~min}$ at $4{ }^{\circ} \mathrm{C}$. The cell pellet was resuspended in $1 \mathrm{~mL}$ of acetone and vigorously

Table 1 Recombinant E. coli cells used in this study

\begin{tabular}{lllll}
\hline Strains & \multicolumn{2}{l}{ Plasmids } & Named in this study \\
\cline { 2 - 3 } & $\mathbf{p A C}-$ Lyc & $\mathbf{p E T}-S I D F$ & $\mathbf{p E T}-$ SIDFG & \\
\hline E. coli K12 & $X$ & & E. coli K12L \\
E. coli BL21 & $X$ & & E. coli BL21L \\
& $X$ & $X$ & & E. coli BL21LF \\
& $X$ & & $X$ & E. coli BL21LG \\
\hline
\end{tabular}


stirred for $10 \mathrm{~min}$ at $4{ }^{\circ} \mathrm{C}$. The mixture was then centrifuged at $10,000 \times g$ for $10 \mathrm{~min}$, and the acetone supernatant was filtered through a $0.2 \mu \mathrm{m}$ nylon sterile filter. Then, samples were lyophilized (Thermo Scientific Heto PowerDry) and the final extract was resuspended in $0.1 \mathrm{~mL}$ of a 50:50 (v:v) mixture of A:B mobile phases for HPLC analysis. The lycopene extracted from the interphase was treated using the same procedure. The organic solvent phase was filtered through a $0.2 \mu \mathrm{m}$ nylon sterile filter and lyophilized.

\section{Lycopene quantification by HPLC}

The HPLC separation was performed on a Shimadzu HPLC equipped with a multi-channel pump (mod LC-20AD) and a DAD detector $(\bmod$ SPD-M20A) with a Develosil ${ }^{\circledR}$ C30-UG-5 column $(250 \mathrm{~mm} \times 4.6 \mathrm{~mm} \times 5 \mu \mathrm{m})$ from Phenomenex. Elution conditions were based on the chromatographic method developed by (Sander et al. 1994) with modifications. Two mobile phases were used: phase A, composed of methanol and water $(96: 4, \mathrm{v}: \mathrm{v})$ and phase B, tert-butyl methyl ether. The flow rate was $1.2 \mathrm{~mL} \mathrm{~min}^{-1}$ and the injection volume $40 \mu \mathrm{L}$. The column was thermostated at $30{ }^{\circ} \mathrm{C}$. The separation of carotenoid standards and extracts was carried out using a linear mobile phase gradient from 50:50 (volume ratio, A/B) to 37:63 (volume ratio, $\mathrm{A} / \mathrm{B}$ ) in $12 \mathrm{~min}$; then the system was restored to its initial condition for $5 \mathrm{~min}$. The concentrations of lycopene, 13-cis-lycopene and phytoene were calculated using response factors relative to the internal standard, $\beta$-apo-8-carotenal. Carotenoid identification was carried out by comparing the retention times and absorption spectra characteristics (Additional file 1: Table S1) by reference to standards purchased from Sigma Aldrich. Detection was performed at $472 \mathrm{~nm}$ for lycopene and 13-cis-lycopene and at $285 \mathrm{~nm}$ for phytoene (Additional file 1: Figure S2). Measurements obtained from cell extracts were compared to curves generated from stand$\operatorname{ards}\left(R^{2}=0.99\right)$.

\section{Transmission electron microscopy (TEM)}

Escherichia coli BL21LF cells and interphase samples were fixed with $3 \%$ glutaraldehyde for $30 \mathrm{~min}$ and prepared as previously described (Huxley and Zubay 1960). The ultrathin sections were cut in a Reichert-Young ultramicrotom. Staining was carried out with $2 \%$ uranyl acetate. Sections were then examined by using a Carl Zeiss EM 10 C electron microscope.

\section{Flow cytometry (FCM)}

Samples were run by flow cytometry (FCM) in a BectonDickinson FASort model equipped with an argon laser for excitation at $488 \mathrm{~nm}$ and $15 \mathrm{~mW}$ and filters at 525 and $630 \mathrm{~nm}$. Samples were adjusted to an event rate of $800-2000$ cells s$^{-1}$ and a total of 10,000 events were registered per sample. To determine cell viability by FCM, double staining was performed accordingly to (Hewitt et al. 1999). PI and BOX were used for viability studies on living cells. The FCM probe fluoresceinpropidium iodide (PI) was purchased from Sigma-Aldrich, while bis-(1,3dibutylbarbituric acid) trimethine oxonol (Bis-oxonol, BOX) was purchased from Molecular Probes Inc. Stained cells were diluted in phosphate buffered saline solution pH 7.2 (PBS). FALS and RALS values allowed cell debris discrimination and a total of 10,000 events were used for statistical data analysis.

Heat stressed cells treated at $60^{\circ} \mathrm{C}$ for $30 \mathrm{~min}$ and exponentially growing cells were used as positive and negative controls, respectively. The green fluorescence channel for BOX-stained cells (X-axis) was plotted versus the red fluorescence channel for PI stained cells (Y-axis). Flow cytometry data were analysed with WinList 5.0 (Verity Software House) software.

\section{Results}

\section{Optimization of IPTG concentration}

Recombinant E. coli BL21LF and E. coli BL21LG (Table 1) were cultivated in MM9 medium containing $20 \mathrm{mM}$ glucose as carbon source with $200 \mathrm{rpm}$ orbital shaking and $28{ }^{\circ} \mathrm{C}$. When the cultures reached $0.5 \mathrm{OD}$, the IPTG inductor was added at concentrations ranging from 0 to $1.0 \mathrm{mM}$. Samples were taken from each culture for lycopene extraction at $24 \mathrm{~h}$, when the lycopene production was maximum. As shown in Fig. 1, a positive correlation between lycopene production and inductor concentration was noted for the two recombinant E. coli BL21 strains. Based on these results, the IPTG concentration selected was 0.1 and $0.4 \mathrm{mM}$ for E. coli BL21LF and $E$. coli BL21LG, respectively.

\section{Optimization of culture conditions}

In order to determine the optimal culture conditions, besides lycopene, the 13-cis-lycopene and phytoene content were quantified, the first since it is a lycopene isomer and is often produced by lycopene oxidative degradation (Chasse et al. 2001) and the second, as the precursor of lycopene. To select a carbon source for lycopene production, $50 \mathrm{~mL}$ batch cultures for the whole recombinant $E$. coli strains (K12L, BL21L, BL21LF and BL21LG) were carried out using either $40 \mathrm{mM}$ glycerol or $20 \mathrm{mM}$ glucose (Additional file 1: Figure S3). All cultures were made by orbital shaking at $200 \mathrm{rpm}$ and $28^{\circ} \mathrm{C}$, which is the optimal temperature for lycopene biosynthesis (Kim et al. 2011). The biomass, lycopene, 13-cis-lycopene and phytoene content were determined at $24 \mathrm{~h}$. The specific growth rate was also calculated for each culture (Table 2). 


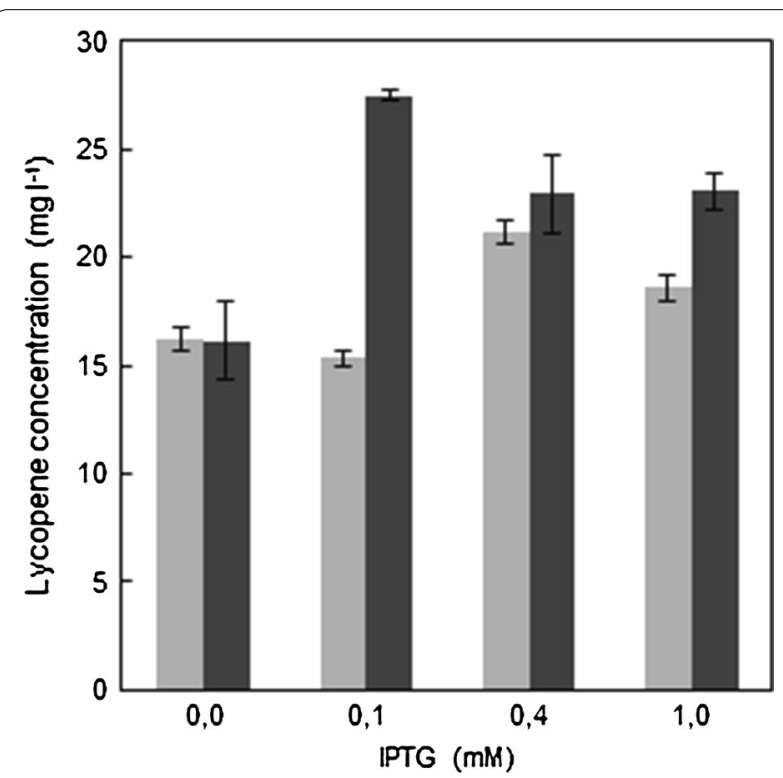

Fig. 1 Lycopene production at different IPTG concentrations after $24 \mathrm{~h}$ in glucose culture. Grey bars and solid bars represent the results obtained using E. coli BL21F and the E. coli BL21G, respectively. Data represent the means and standard deviations from three separate experiments

Table 2 Effect of carbon source on cell growth (OD), lycopene production and specific growth rate $\left(\mu_{\max }\right)$ in the $E$. coli aqueous cultures

\begin{tabular}{llll}
\hline Carbon source & OD $(600 \mathbf{n m})$ & Lycopene $\left(\mathbf{m g ~ L}^{-\mathbf{1}}\right)$ & $\boldsymbol{\mu}_{\mathbf{m a x}}\left(\mathbf{h}^{-\mathbf{1}}\right)$ \\
\hline Glucose & & & \\
E. coli K12L & $3.56 \pm 0.07$ & $16.18 \pm 0.81$ & $0.33 \pm 0.03$ \\
E. coli BL21L & $3.86 \pm 0.04$ & $11.51 \pm 0.52$ & $0.43 \pm 0.01$ \\
E. coli BL21LF & $4.27 \pm 0.04$ & $27.51 \pm 1.35$ & $0.43 \pm 0.02$ \\
E. coli BL21LG & $3.29 \pm 0.17$ & $21.15 \pm 1.01$ & $0.35 \pm 0.04$ \\
Glycerol & & & \\
E. coli $\mathrm{K} 12 \mathrm{~L}$ & $3.61 \pm 0.15$ & $20.88 \pm 1.04$ & $0.27 \pm 0.01$ \\
E. coli BL21L & $3.95 \pm 0.24$ & $14.84 \pm 0.74$ & $0.30 \pm 0.04$ \\
E. coli BL21LF & $2.79 \pm 0.04$ & $37.56 \pm 1.41$ & $0.31 \pm 0.01$ \\
E. coli BL21LG & $1.55 \pm 0.06$ & $27.28 \pm 0.81$ & $0.28 \pm 0.06$ \\
\hline
\end{tabular}

Data represent the means and standard deviations from three separate experiments

Glycerol cultures exhibited the highest lycopene production, whereas glucose cultures showed the highest cell mass and specific growth rate. In all cultures, the phytoene concentration was very low compared with lycopene (lower than $5 \%$ ), demonstrating a optimal expression of pAC-Lyc plasmid (Rodriguez-Villalon et al. 2008). The maximum lycopene content was obtained from the Ecoli BL21LF strain when glycerol was used as carbon source (Fig. 2). Therefore, glycerol was selected

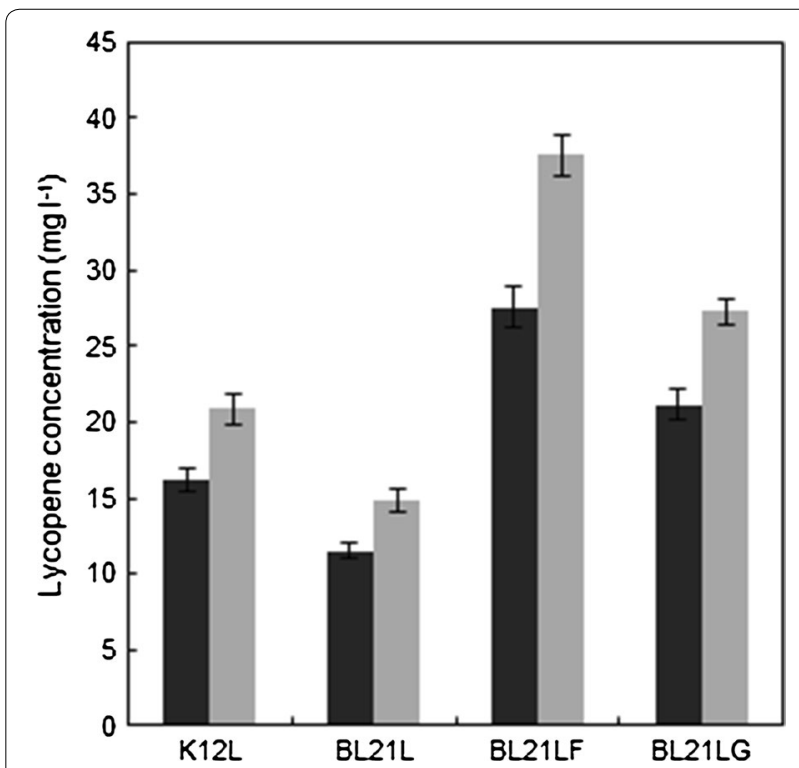

Fig. 2 Comparison between glucose (solid bars) and glycerol (grey bars) as main carbon sources for lycopene production after $24 \mathrm{~h}$ using the recombinant E. coli strains. Data represent the means and standard deviations from three separate experiments

as carbon source for lycopene production with $E$. coli BL21LF in all subsequent studies.

The effect of key fermentation control parameters, such as shaking speed and light, on the production of lycopene were also tested. In the whole set of cultures exposed to light, the concentration of 13-cis-lycopene was very low compared with lycopene (lower than $8 \%$ ). When these same cultures were grown in darkness, no differences were detected for the isomer concentration, thus this additional precaution was discarded (data not shown). Orbital shaking speed was investigated as a putative bioreactor parameter responsible for controlling dissolved oxygen content and maximum cell density. Batch cultures in $500 \mathrm{~mL}$ flasks with $50 \mathrm{~mL}$ MM 9 medium were carried out at three orbital shaking speeds: 100, 200 and 400 rpm. However, there were no differences in lycopene production.

Lycopene production was evaluated in detail using glycerol as carbon source and the selected $E$. coli BL21LF in $50 \mathrm{~mL}$ batch culture and with $200 \mathrm{rpm}$ orbital shaking at $28{ }^{\circ} \mathrm{C}$. For this purpose, three samples were taken at different times up to $24 \mathrm{~h}$ (Fig. 3). Lycopene production started during the exponential growth phase, and the maximum lycopene concentration was reached in the stationary phase, since it is a secondary metabolite biomass dependent.

\section{Increase of lycopene production via in situ extraction in organic-aqueous culture systems}

The effect of organic solvents on cell growth and lycopene production in metabolically engineered $E$. coli 


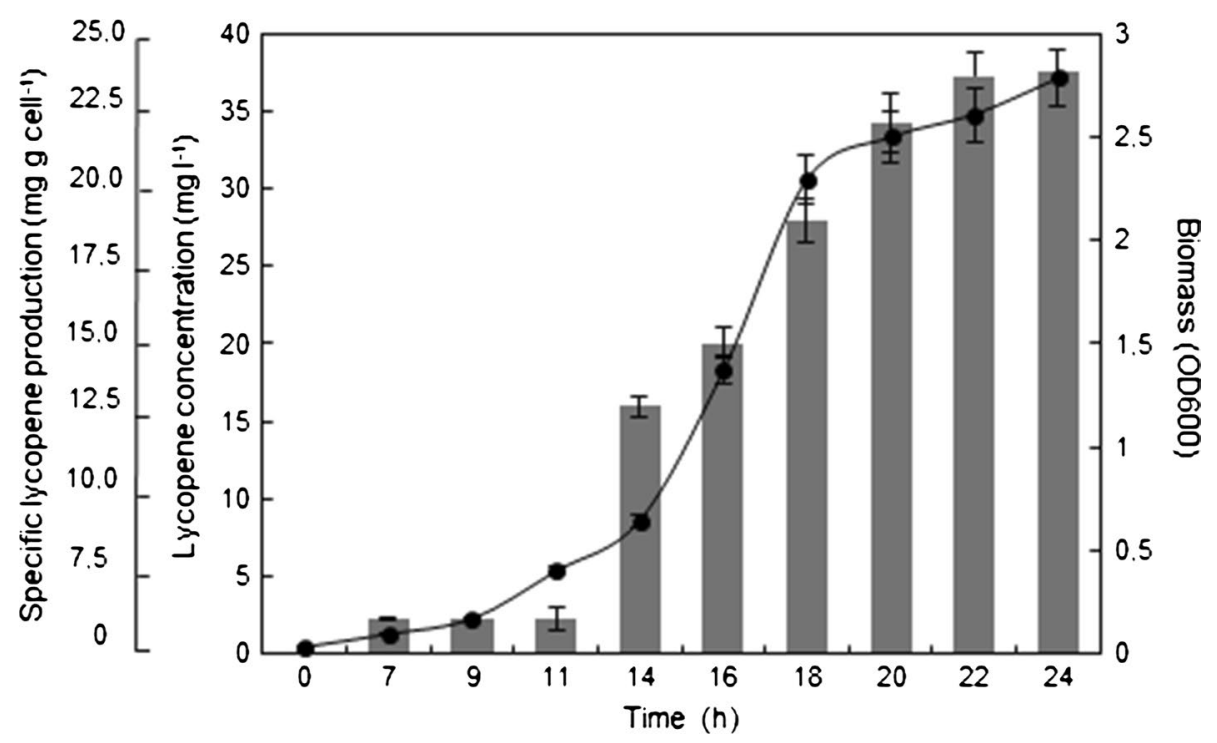

Fig. 3 Specific lycopene content (grey bars) and biomass (filled circle) of recombinant E. coli BL21LF in $50 \mathrm{~mL}$ batch culture with $40 \mathrm{mM}$ glycerol, $200 \mathrm{rpm}$ and $28^{\circ} \mathrm{C}$. Data represent the means and standard deviations from three separate experiments

BL21LF was investigated in batch cultures. Organic solvents for lycopene extraction were selected as a function of the $\log$ P solvent, which ranged from $3.76,4.27,4.78$, 5.8 to 6.31 for heptane, hexane, octane, decane, undecane and dodecane, respectively.

Batch cultures were performed using an organic:culture broth volume ratio of $1: 5(\mathrm{v} / \mathrm{v})$ for the whole set of the $50 \mathrm{~mL}$ cultures. Organic solvent was added when the biomass reached 0.5 OD, in order not to affect cell growth. Hexane and heptane were rejected; hexane due to its high volatility and heptane as a consequence of its toxicity to cells. An aqueous culture without organic solvent was used as control system and the resulting lycopene production $\left(37.56 \pm 1.41 \mathrm{mg} \mathrm{L}^{-1}\right)$ was used to normalize lycopene biosynthesis.

In all the aqueous-organic systems, three phases were visible after $24 \mathrm{~h}$ in the presence of organic solvent: an aqueous phase containing the $E$. coli BL21LF cells, an interphase and the organic phase. This tri-phasic culture system and normalized lycopene production at $24 \mathrm{~h}$ for all the organicaqueous culture systems can be seen in Fig. 4A, B. Interestingly, lycopene production greatly increased with the addition of organic solvent except for undecane, although the lycopene production profile presented a negative correlation with the solvent $\log$ P. Final lycopene production after $24 \mathrm{~h}$ was $198.9,148.6$ and $115.97 \%$ for octane, decane and dodecane, respectively, with respect to the control aqueous culture. In these organic-aqueous culture systems, lycopene was extracted from the cells to the interphase and the organic phase, which greatly enhanced production. Maximum lycopene production was obtained for the octane-aqueous system reaching $74.71 \pm 3.74 \mathrm{mg} \mathrm{L}^{-1}$ (198.9\%). This lycopene production was distributed among the three phases formed: $12.24 \pm 0.61 \mathrm{mg} \mathrm{L}^{-1}$ into $E$. coli BL21LF cells of the aqueous phase, $57.14 \pm 2.85 \mathrm{mg} \mathrm{L}^{-1}$ in the interphase and $5.32 \pm 0.24 \mathrm{mg} \mathrm{L}^{-1}$ in the octane phase. As regards to the cell density, the OD at $600 \mathrm{~nm}$ of the aqueous media was similar to the aqueous-organic systems at $24 \mathrm{~h}$, around $4.00 \pm 0.35$. The E. coli BL21LF growth culture is shown in Additional file 1: Figure S4.

In order to study the physicochemical properties of the interphase formed in the aqueous-organic systems, transmission electron microscopy (TEM) was used (Fig. 5A). Spherical particles of homogeneous size ranging from 15 to $20 \mathrm{~nm}$ were observed, demonstrating its proteo-lipidic nature and with high lycopene storage capacity of these particles, representing $76.5 \%\left(57.14 \pm 2.85 \mathrm{mg} \mathrm{L}^{-1}\right)$ of the total culture content. TEM was also used to visualize E. coli BL21LF cells after $24 \mathrm{~h}$ (Fig. 5B-F). Pictures b and c show $E$. coli BL21LF cells in the aqueous medium, whereas d and e show E. coli BL21F cell within the octane-aqueous culture system, and f shows $E$. coli BL21LF in the heptane-aqueous culture system. As depicted, E. coli BL21LF cell growth in aqueous media entirely maintained the outer membrane structure, and even the lipid double layer could be observed. But when the octane-aqueous culture system was employed, the cells partially lost the structural integrity of their outer membrane. This explains the high extractive capacity of octane for lycopene and the proteo-lipidic interphase formation. Cells completely lost their structural integrity when heptane was used. 

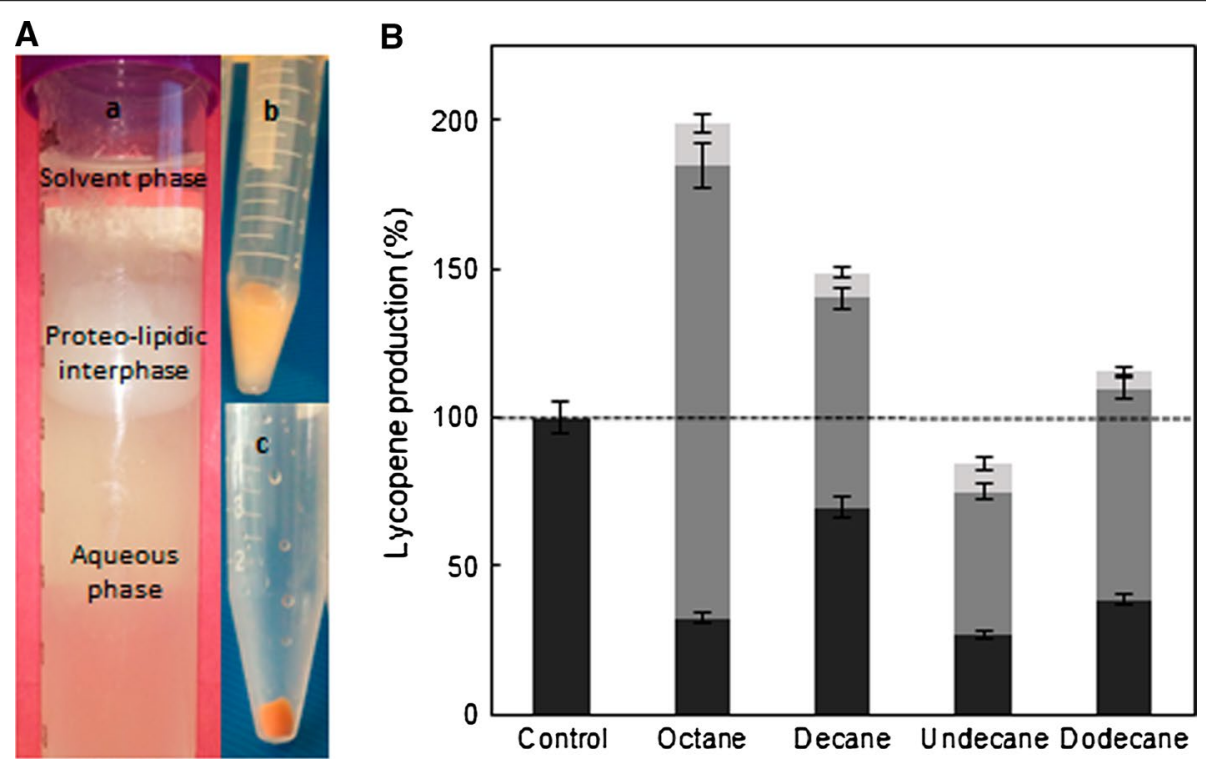

Fig. 4 A Tri-phasic culture system for aqueous-organic systems. $b$ Interphase. c E. coli BL21LF cells. Both phases were orange by the presence of lycopene. B Lycopene production normalized at $24 \mathrm{~h}$ for the organic-aqueous culture systems with E. coli BL21LF, distributed among three phases: aqueous phase containing E. coli BL21LF cells (solid bars), interphase (grey bars) and organic phase (open bars)
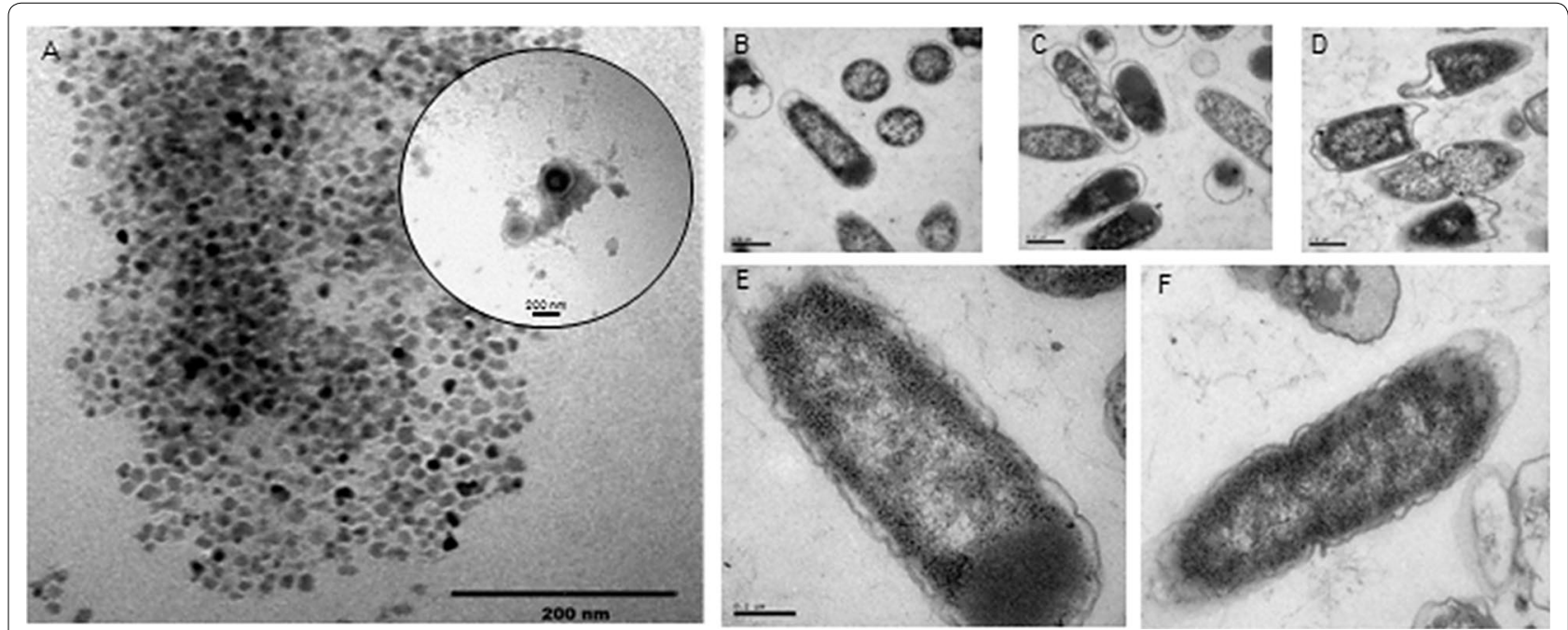

Fig. 5 A Transmission electron microscopy of the interphase of the octane-aqueous culture prepared with negative staining. Transmission electron microscopy of E. coli BL21LF: B and $\mathbf{C}$ in aqueous media, $\mathbf{D}$ and $\mathbf{E}$ in aqueous-octane culture system and $\mathbf{F}$ in aqueous-heptane culture system. Amplifications were $\times 40,000$ for B, C and $\mathbf{D}$ and $\times 100,000$ for $\mathbf{E}$ and $\mathbf{F}$

To determine cell viability, flow cytometry (FCM) using scattered light was chosen. Two fluorochromes were employed simultaneously: bis-(1,3-dibutylbarbituric acid) trimethine oxonol (Bis-oxonol, BOX) and propidium iodide (PI). BOX is a lipophilic anionic compound, which accumulates intracellularly when the cytoplasmic membrane is depolarised, while PI binds to DNA, but cannot cross an intact cytoplasmic membrane (Hewitt et al. 1999). Cell analysis by FCM demonstrated that, during a fed-batch culture in an aqueous-octane system, there was a gradual change in the physiological state of $E$. coli BL21LF. From samples taken at $24 \mathrm{~h}$, three main subpopulations of cells were observed (Fig. 6). These populations corresponded to healthy unstained cells (A3); cells with a depolarised cytoplasmic membrane (A4), stained with BOX; and dead cells with permeabilised membranes, namely cells stained with both PI and BOX (A2). From Fig. 6, the cell number and the percentage within 


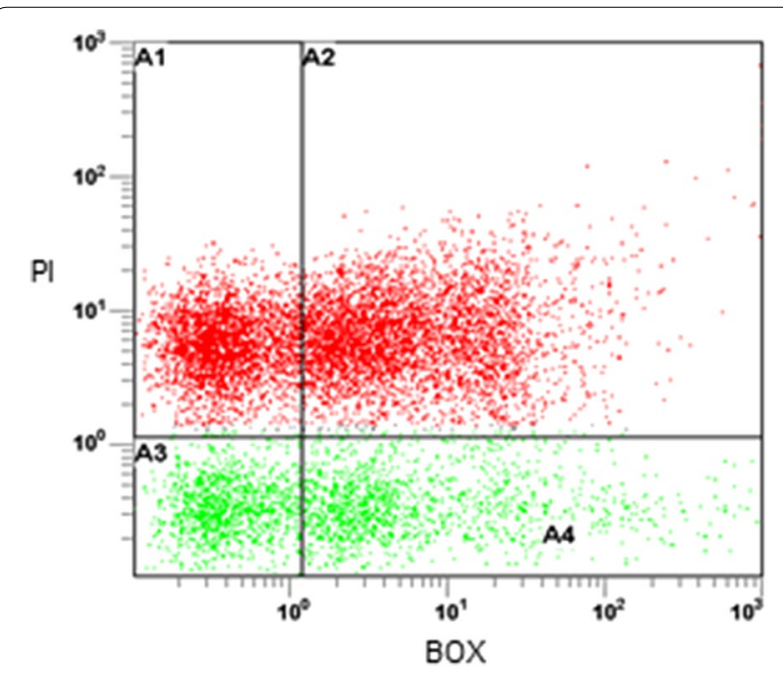

Fig. 6 Flow cytometry (FCM) of the E. coli BL21LF strains in an aqueous-octane culture system. Green fluorescence of cells (axis $\mathrm{X}$ ) due to $\mathrm{BOX}$ is plotted versus red fluorescence (axis $\mathrm{Y}$ ) due to $\mathrm{PI}$. Samples were taken from the batch reactor at $24 \mathrm{~h}$

each quadrant was determined using the CF software. The results are shown in Table 3. Cells from a microbial culture can be grouped according to their different metabolic states and/or extent of cell integrity: (a) intact and metabolically active cells, showing growth capacity, (b) depolarized cells, unable to maintain their intact membrane potential, but that can be recovered temporarily (Cánovas et al. 2007), (c) and dead cells with permeabilised and depolarised membrane (Hewitt et al. 1999). The aqueous-octane culture system $E$. coli BL21LF after $24 \mathrm{~h}$, resulted in an $11.5 \%$ of healthy unstained cells and a $13.24 \%$ of deporalised but recoverable BOX stained cells. Therefore, both of them $(24.74 \%)$ can be considered viable for the continuous production of lycopene.

\section{Aqueous-octane culture system for in situ extraction and semi-continuous lycopene production}

Maximum lycopene production was obtained from the octane-aqueous system (1:5 volume ratio) after $24 \mathrm{~h}$ of culture, when cell growth had ceased and the lycopene content was distributed among the three phases. From

Table 3 Cells number and percentage in each one of the FCM windows

\begin{tabular}{lcc}
\hline Window & Cells number & Percentage (\%) \\
\hline A1 & 179 & 1.79 \\
A2 & 7347 & 73.47 \\
A3 & 1150 & 11.50 \\
A4 & 1324 & 13.24 \\
\hline
\end{tabular}

Cell debris was identified on the basis of the FALS and RALS values these results, a series batch reactor was designed for lycopene extraction, as depicted in Fig. 7a. The aqueousoctane culture system was maintained in operation for $24 \mathrm{~h}$, then the volume of the aqueous phase corresponding to a final 0.05 OD was used as the inoculum for a second batch reactor (taking into account the viable cells fraction). This process was repeated 5 times.

Figure $7 \mathrm{~b}$ displays the lycopene production distributed in each phase and cycle. The lycopene produced in the first cycle was used to normalize the lycopene content of the remaining cycles. In the first three, the lycopene production was $74.71 \pm 3.74 \mathrm{mg} \mathrm{L}^{-1}$, $51.03 \pm 2.51 \mathrm{mg} \mathrm{L}^{-1}$ and $46.45 \pm 2.13 \mathrm{mg} \mathrm{L}^{-1}$, respectively, all of them higher than that obtained in the aqueous medium (37.56 $\left.\pm 1.41 \mathrm{mg} \mathrm{L}^{-1}\right)$. Lycopene production was $29.01 \pm 1.52 \mathrm{mg} \mathrm{L}^{-1}$ and $36.96 \pm 1.82 \mathrm{mg} \mathrm{L}^{-1}$ for the fourth and fifth cycle, respectively, similar to that obtained in the aqueous medium. The biomass reached in each batch reactor was similar, about $3.82 \pm 0.35 \mathrm{OD}$, although production deceased with each cycle.

\section{Discussion}

Metabolic engineering to increase lycopene production in $E$. coli has previously focused on overexpression of the several key isoprenoid genes (Alper et al. 2005). Hence, the first part of this study was focused on the optimization of both the recombinant E. coli strain and culture conditions. When the recombinant E. coli BL21LF and E. coli BL21G strains were used in combination with the IPTG-induced, lycopene production increased, although excessive IPTG concentration reduced lycopene biosynthesis (Fig. 1). This finding is consistent with previous observations of the induction inhibitory effects of lycopene production (Kim and Keasling 2001; Rodriguez-Villalon et al. 2008; Yoon et al. 2008) since, a high induction could cause a shortage of the available precursors for the essential metabolic roles. The IPTG concentrations selected were 0.1 and $0.4 \mathrm{mM}$ for $E$. coli BL21LF and $E$. coli BL21LG, respectively. The lower lycopene production obtained with E. coli BL21LG was probably due to the high energy cost of the extra isp $\mathrm{G}$ gene overexpression, which encodes the HDS protein. It has been revealed that isp $G$ gene basal expression is higher than the expression of other of isoprenoid genes under normal growth conditions (Yuan et al. 2006). Accordingly, a high multi-copy expression vector may cause a metabolic imbalance. In addition, the HDS enzyme (4-hydroxy-2-methyl-2-butenyl 4-diphosphate synthase) flavodoxin reductase and NADPH dependent metalloprotein, (Rohdich et al. 2003; Seemann et al. 2006) involve an extra expense of reducing power to carry out their catalytic functions (Hunter 2007). These assumptions could explain the E. coli BL21LG strain delay metabolic. 

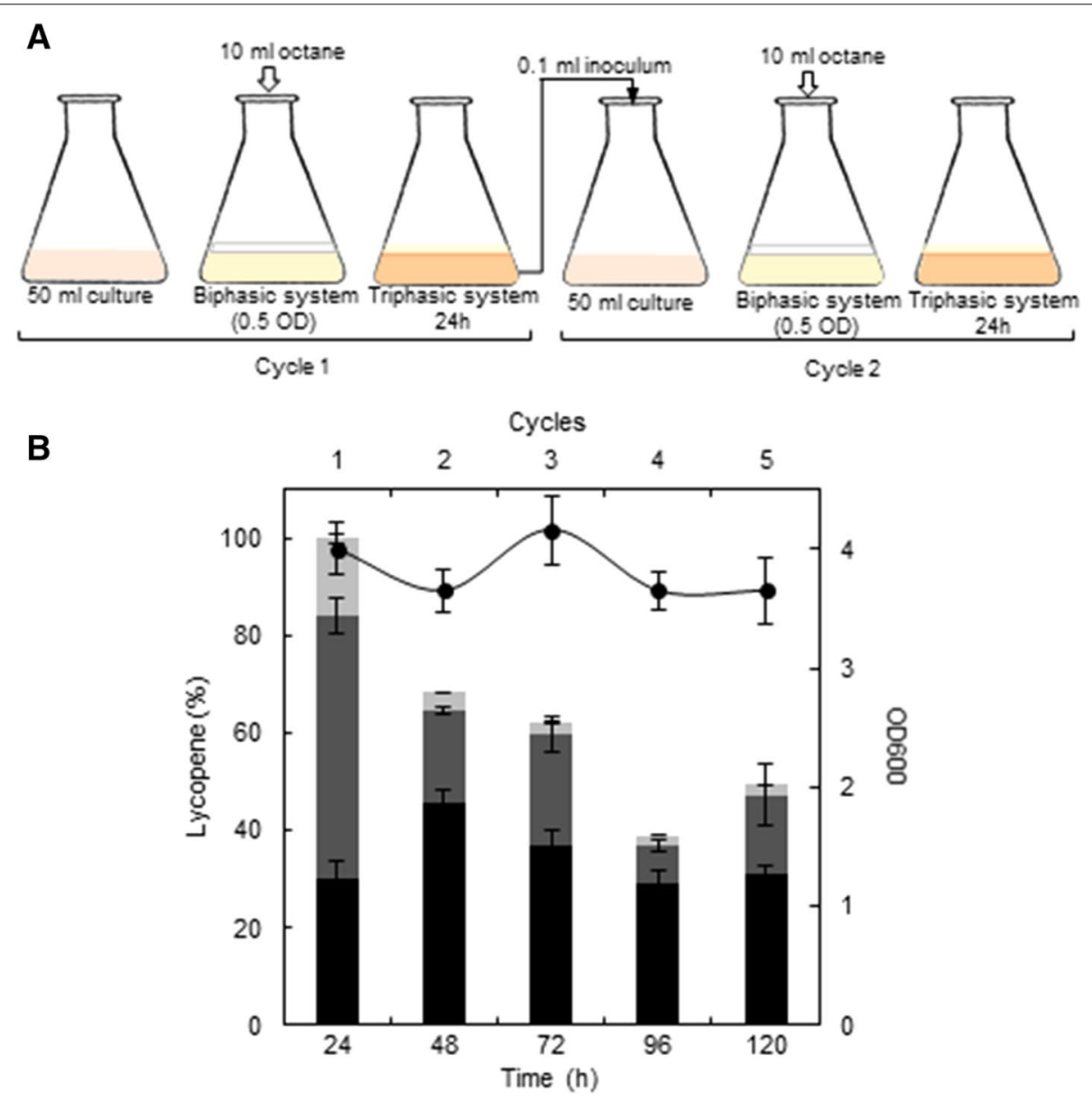

Fig. 7 a Series batch reactor for lycopene over-production using E. coli BL21LF in an aqueous-octane culture system. b Normalized lycopene production distributed within each phase for each cycle: aqueous phase containing E. coli BL21LF cells (solid bars), interphase (grey bars) and organic phase (light grey bars), and biomass (filled circle). Data represent the means and standard deviations from three separate experiments

In previous studies, glycerol and glucose were compared as carbon sources for secondary metabolite production, glycerol being seen as a better carbon source than glucose (Lee 1996; Martin et al. 2001; Yoon et al. 2009). Glycerol reduces cell growth, but stimulates metabolite production (Fang and Demain 1997).

On the other hand, the recombinant E. coli BL21 and K12 strains showed differences in lycopene production, the latter producing 1.4-fold more than the E. coli BL21L strain (Fig. 2). Significant differences have been demonstrated at gene transcription and metabolomic profile levels between both E. coli strains (Alper et al. 2006). In addition, the outer membranes of E. coli $\mathrm{K} 12$ and $E$. coli BL21 also show differences in lipoprotein and lipid composition, which may influence cell envelope permeability and integrity. These differences could affect potential lycopene accumulation (Yoon et al. 2012; Marisch et al. 2013).
The maximum lycopene production and specific lycopene production were $37.56 \pm 1.41 \mathrm{mg} \mathrm{L}^{-1}$ and $25.34 \pm 1.2 \mathrm{mg} \mathrm{g} \mathrm{cell}^{-1}$, respectively, which was obtained during stationary growth phase using $E$. coli BL21LF in $40 \mathrm{mM}$ glycerol and $0.1 \mathrm{mM}$ IPTG (Fig. 2). This production is excellent compared with the lycopene levels previously reported. Stephanopoulos group's (2006) created a triple knockout strain, $\Delta g d h \mathrm{~A} \Delta a c e \mathrm{E} \Delta f d h \mathrm{~F}$, which exhibited a lycopene production of $8.15 \mathrm{mg} \mathrm{g}$ cell $^{-1}$ (Alper et al. 2006). Kin et al. (2011) used a metabolically engineered $E$. coli strain, reporting a lycopene production of $32 \mathrm{mg} \mathrm{g}$ cell $^{-1}$ in fed-batch cultures with glycerol supplemented with glucose as auxiliary carbon source. Recently, a higher level of lycopene production (33.43 $\mathrm{mg} \mathrm{g} \mathrm{cell}^{-1}$ ) was attained by native appY promoter replacement of a $\mathrm{T} 5$ promoter, and the deletion of the iclR gene in E. coli CBW 12241 (Chen et al. 2013). High lycopene production was also achieved $(18.49 \mathrm{mg} \mathrm{g}$ 
cell $^{-1}$ ) using a CRP engineering strategy (Huang et al. 2015).

One of the main limitations of biotechnological lycopene production is the fact that it is stored as an intracellular product in the membrane (Fraser and Sandmann 1992). Therefore, special must be taken into account to identify the optimal parameters for continuous lycopene production. It has been assumed that the upper limit for the carotenoid production in a non-carotenogenic $E$. coli is around $2 \mathrm{mg} \mathrm{g}$ cell $^{-1}$ due to the limited lipophilic carotenoid storage capacity of the membrane (Albrecht et al. 1999; Sandmann 2001). Hence, a new strategy is needed to overcome the lycopene accumulation barrier. Optimal parameters must be determined in order to promote lycopene production during both the exponential and stationary phase, while stimulating in situ extraction to prevent accumulation in the cell membrane. Moreover, when the whole cells are employed as biocatalyst, productivity may decrease due to end-product inhibition or accumulation: however, if the product is continuously removed by a solvent phase, an increase in activity/productivity can be attained. To achieve this aim, a two-phase culture system using an organic solvent was proposed to maximize the lycopene production through in situ extraction from the cells. Few studies using organic solvents for terpenes extraction have been published. It has been reported a two-phase culture system with dodecane for retinoids extraction using a metabolically engineered E. coli (Jang et al. 2011). In a previous report, a two-phase culture system with decane and $0.1 \%\left(\mathrm{w} \mathrm{v}^{-1}\right)$ Span 20 was successfully applied for lycopene production $\left(9.6 \pm 1.0 \mathrm{mg} \mathrm{g}^{-1}\right)$ (Yoon et al. 2008). However, lycopene was inefficiently extracted from the recombinant $E$. coli strain without partial digestion of the cell wall with lysozyme. The authors used E. coli spheroplasts in order to increase the extraction, but their instability reduced the possibility of designing a continuous system.

Another aspect to consider in a biphasic system is the organic solvent toxicity toward microorganisms. This toxicity depends on its inherent toxicity and the intrinsic tolerance of the bacterial species and strains (Ramos et al. 2002). The toxicity of a solvent correlates with the logarithm of its partition coefficient in n-octanol and water $(\log \mathrm{P})$, meaning that, organic solvents with a $\log \mathrm{P}$ of between 1.5 and 4.0 are toxic for microorganisms. Six organic solvents were selected for lycopene extraction as a function of the log P, ranging from 3.76 to 6.31. In all aqueous-organic systems tested in this study three phases were formed after $2 \mathrm{~h}$ of culture: an aqueous phase containing cells, an interphase and an organic phase (Fig. 4A). Lycopene was removed from the cells to the interphase and the organic phase, thus enhancing production. Maximum lycopene production was obtained from octane-aqueous systems $(5: 1, \mathrm{v}$ $\left.\mathrm{v}^{-1}\right)\left(74.71 \pm 3.74 \mathrm{mg} \mathrm{L}^{-1}\right.$ or $\left.49.70 \pm 2.48 \mathrm{mg} \mathrm{g} \mathrm{cell}^{-1}\right)$, a 2-fold improvement over that attained in aqueous culture. This production was also much higher than that obtained from tomato, $0.42 \mathrm{mg} \mathrm{g}^{-1}$, which suggest it is a promising strategy for its industrial production (Sharma and Le Maguer 1996). This lycopene production rate is, to our knowledge, the highest reported in the literature to date. Moreover, the proteo-lipidic nature of the interphase demonstrated by TEM (Fig. 5A), showed a high lycopene storage capacity of $76.5 \%\left(57.15 \pm 2.86 \mathrm{mg} \mathrm{L}^{-1}\right)$ with respect to the total production of lycopene in aqueous-octane systems (Fig. 4B). This interface was formed from partial outer membrane disintegration, while cells with structural integrity were found in the aqueousoctane systems after $24 \mathrm{~h}$ (Fig. 5D, E). Additionally, flow cytometry analysis of E. coli BL21LF cells showed a significant percentage $(24.74 \%)$ of viable and cultivatable cells for continuous lycopene production (Fig. 6; Table 3). From these results, a series batch reactor for semi-continuous lycopene extraction was designed (Fig. 7a). The biomass reached was similar for the all cycles, although lycopene production decreased with each of cycle. The results further support the idea that cell depolarisation indicates a decline in cell functionality due to energy depletion, but does not involve cell death. Besides, lycopene accumulation in the cell membrane seems to affect lycopene biosynthesis, since the amount of lycopene extracted from cells to the interphase and the organic phase decreased in each cycle. Nevertheless, the lycopene production obtained from the fifth cycle $(120 \mathrm{~h})$ was $36.96 \pm 1.82 \mathrm{mg} \mathrm{L}^{-1}$, similar to that obtained in the aqueous medium (Fig. 7b).

In this study, semi-continuous lycopene overproduction and in situ extraction using a metabolically engineered E. coli strain is attained for the first time with an octane-aqueous culture system (1:5 volume ratio). In the future, we hope these findings will be useful for industry and constitute an important step forward in the development of a competitive biotechnological lycopene production system.

\section{Additional file}

Additional file 1: Table S1. Retention times and absorption spectra characteristics of carotenoids. Figure S1. Biosynthetic pathway of lycopene in Escherichia coli from a native 2-C-methyl-D-erythritol 4-phosphate pathway (non-mevalonate pathway). Gene names and its encoded enzymes follow: $d x r$ DXP reductoisomerase, $d x s$ DXP synthase, idi IPP isomerase, ispA FPP synthase, crtE GGPP synthase, crtB phytoene synthase, $\mathrm{crt} /$ phytoene desaturase. Figure S2. Chromatogram of reference standards for 20 min measured at 472 and 285 nm, respectively. Figure S4. $E$. coli BL21 LF growth in the presence of octane during $24 \mathrm{~h}$. 


\section{Authors' contributions}

TDP and JGJ conceived and coordinated the study and wrote the paper. TDP, $J G J$ and AR designed, performed and analyzed all experiments in paper. AE-C, $A M$, and $M C$ reviewed the results and approved the final version of the manuscript. All authors read and approved the final manuscript.

\section{Acknowledgements}

The authors thank Prof. G. Stephanopoulos (Department of Chemical Engineering, Institute of Technology, Cambridge, Massachusetts, EEUU) for providing plasmids used in this work. This work was supported by a Grant (BIO2014-54411-C2-1-R) from Ministry of Science and Innovation and partially by Seneca Foundation CARM 19236/PI/14.

\section{Compliance with ethical guidelines}

\section{Competing interests}

The authors declare that they have no competing interests.

Received: 23 June 2015 Accepted: 3 September 2015

Published online: 22 September 2015

\section{References}

Albrecht M, Misawa N, Sandmann G (1999) Metabolic engineering of the terpenoid biosynthetic pathway of Escherichia coli for production of the carotenoids beta-carotene and zeaxanthin. Biotechnol Lett 21:791-795. doi:10.1023/A:1005547827380

Alper H, Miyaoku K, Stephanopoulos G (2005) Construction of lycopeneoverproducing E. coli strains by combining systematic and combinatorial gene knockout targets. Nat Biotechnol 23:612-616. doi:10.1038/nbt1083

Alper H, Miyaoku K, Stephanopoulos G (2006) Characterization of lycopeneoverproducing E. coli strains in high cell density fermentations. Appl Microbiol Biotechnol 72:968-974. doi:10.1007/s00253-006-0357-y

Araya-Garay JM, Feijoo-Siota L, Rosa-Dos-Santos F, Veiga-Crespo P, Villa TG (2012) Construction of new Pichia pastoris X-33 strains for production of lycopene and beta-carotene. Appl Microbiol Biotechnol 93:2483-2492. doi:10.1007/s00253-011-3764-7

Baba T, Ara T, Hasegawa M, Takai Y, Okumura Y, Baba M, Datsenko KA, Tomita M, Wanner BL, Mori H (2006) Construction of Escherichia coli K-12 inframe, single-gene knockout mutants: the Keio collection. Mol Syst Biol 2(2006):0008. doi:10.1038/msb4100050

Bahieldin A, Gadalla NO, Al-Garni SM, Almehdar H, Noor S, Hassan SM, Shokry AM, Sabir JSM, Murata N (2014) Efficient production of lycopene in Saccharomyces cerevisiae by expression of synthetic crt genes from a plasmid harboring the ADH2 promoter. Plasmid 72:18-28. doi:10.1016/j. plasmid.2014.03.001

Cánovas M, García V, Bernal V, Torroglosa T, Iborra JL (2007) Analysis of Escherichia coli cell state by flow cytometry during whole cell catalyzed biotransformation for I-carnitine production. Process Biochem 42:25-33. doi:10.1016/j.procbio.2006.07.027

Chasse G, Mak ML, Deretey E, Farkas I, Torday LL, Papp JG, Sarma DSR, Agarwal A, Chakravarthi S, Agarwal S, Rao AV (2001) An ab initio computational study on selected lycopene isomers. J Mol Struct THEOCHEM 571:27-37. doi:10.1016/S0166-1280(01)00424-9

Chen Y-Y, Shen H-J, Cui Y-Y, Chen S-G, Weng Z-M, Zhao M, Liu J-Z (2013) Chromosomal evolution of Escherichia coli for the efficient production of lycopene. BMC Biotechnol 13:6. doi:10.1186/1472-6750-13-6

Fang A, Demain A (1997) Influence of aeration and carbon source on production of microcin B17 by Escherichia coli ZK650. Appl Microbiol Biotechnol 47:547-553

Fraser PD, Sandmann G (1992) In vitro assays of three carotenogenic membrane-bound enzymes from Escherichia coli transformed with different crt genes. Biochem Biophys Res Commun 185:9-15. doi:10.1016/ S0006-291X(05)80947-7

Giovannucci E, Rimm EB, Liu Y, Stampfer MJ, Willett WC (2002) A prospective study of tomato products, lycopene, and prostate cancer risk. J Natl Cancer Inst 94:391-398

Hewitt CJ, Nebe-Von Caron G, Nienow AW, McFarlane CM (1999) Use of multi-staining flow cytometry to characterise the physiological state of Escherichia coli W3110 in high cell density fed-batch cultures. Biotechnol Bioeng 63:705-711. doi:10.1002/ (SICI) 1097-0290(19990620)63:6<705:AID-BIT8>3.0.CO;2-M

Huang L, Pu Y, Yang X, Zhu X, Cai J, Xu Z (2015) Engineering of global regulator CAMP receptor protein (CRP) in Escherichia coli for improved lycopene production. J Biotechnol 199:55-61. doi:10.1016/j.jbiotec.2015.02.006

Hunter WN (2007) The non-mevalonate pathway of isoprenoid precursor biosynthesis. J Biol Chem 282:21573-21577. doi:10.1074/jbc.R700005200

Huxley H, Zubay G (1960) Electron microscope observations on the structure of microsomal particles from E. coli. J Mol Biol 2:10-18

Jang H-J, Yoon S-H, Ryu H-K, Kim J-H, Wang C-L, Kim J-Y, Oh D-K, Kim S-W (2011) Retinoid production using metabolically engineered Escherichia coli with a two-phase culture system. Microb Cell Fact 10:59. doi:10.1186/1475-2859-10-59

Jin YS, Stephanopoulos G (2007) Multi-dimensional gene target search for improving lycopene biosynthesis in Escherichia coli. Metab Eng 9:337-347. doi:10.1016/j.ymben.2007.03.003

Kim S, Keasling JD (2001) Nonmevalonate isopentenyl diphosphate synthesis pathway in Escherichia coli enhances lycopene production. Biotechnol Bioeng 72:408-415

Kim YS, Lee JH, Kim NH, Yeom SJ, Kim SW, Oh DK (2011) Increase of lycopene production by supplementing auxiliary carbon sources in metabolically engineered Escherichia coli. Appl Microbiol Biotechnol 90:489-497. doi:10.1007/s00253-011-3091-z

Kitade Y, Watanabe S, Masaki T, Nishioka M, Nishino H (2002) Inhibition of liver fibrosis in LEC rats by a carotenoid, lycopene, or a herbal medicine, Shosaiko-to. Hepatol Res 22:196-205. doi:10.1016/S1386-6346(01)00132-2

Lee SY (1996) High cell-density culture of Escherichia coli. Trends Biotechnol 14:98-105. doi:10.1016/0167-7799(96)80930-9

Marisch K, Bayer K, Scharl T, Mairhofer J, Krempl PM, Hummel K, Razzazi-Fazeli E, Striedner G (2013) A comparative analysis of industrial Escherichia coli K-12 and B strains in high-glucose batch cultivations on process-, transcriptome- and proteome level. PLoS One. doi:10.1371/journal. pone.0070516

Martin VJJ, Yoshikuni Y, Keasling JD (2001) The in vivo synthesis of plant sesquiterpenes by Escherichia coli. Biotechnol Bioeng 75:497-503. doi:10.1002/bit.10037

Martin VJJ, Pitera DJ, Withers ST, Newman JD, Keasling JD (2003) Engineering a mevalonate pathway in Escherichia coli for production of terpenoids. Nat Biotechnol 21:796-802. doi:10.1038/nbt833

Rabi T, Gupta S (2008) Dietary terpenoids and prostate cancer chemoprevention. Front Biosci 13:3457-3469. doi:10.2741/2940

Ramos JL, Duque E, Gallegos M-T, Godoy P, Ramos-Gonzalez MI, Rojas A, Teran W, Segura A (2002) Mechanisms of solvent tolerance in gramnegative bacteria. Annu Rev Microbiol 56:743-768. doi:10.1146/annurev. micro.56.012302.161038

Rao A (2002) Lycopene, tomatoes, and the prevention of coronary heart disease. Exp Biol Med 227:908-913

Rodriguez-Villalon A, Perez-Gil J, Rodriguez-Concepcion M (2008) Carotenoid accumulation in bacteria with enhanced supply of isoprenoid precursors by upregulation of exogenous or endogenous pathways. J Biotechnol 135:78-84

Rohdich F, Zepeck F, Adam P, Hecht S, Kaiser J, Laupitz R, Gräwert T, Amslinger S, Eisenreich W, Bacher A, Arigoni D (2003) The deoxyxylulose phosphate pathway of isoprenoid biosynthesis: studies on the mechanisms of the reactions catalyzed by IspG and IspH protein. Proc Natl Acad Sci USA 100:1586-1591. doi:10.1073/pnas.0337742100

Sander LC, Sharpless KE, Craft NE, Wise SA (1994) Development of engineered stationary phases for the separation of carotenoid isomers. Anal Chem 66:1667-1674. doi:10.1021/ac00082a012

Sandmann G (2001) Carotenoid biosynthesis and biotechnological application. Arch Biochem Biophys 385:4-12. doi:10.1006/abbi.2000.2170

Sauer U, Lasko DR, Fiaux J, Hochuli M, Glaser R, Szyperski T, Wüthrich K, Bailey JE (1999) Metabolic flux ratio analysis of genetic and environmental modulations of Escherichia coli central carbon metabolism. J Bacteriol 181:6679-6688

Sedjo RL, Roe DJ, Abrahamsen M, Harris RB, Craft N, Baldwin S, Giuliano AR (2002) Vitamin A, carotenoids, and risk of persistent oncogenic human papillomavirus infection. Cancer Epidem Biomarkers Prev 11:876-884

Seemann M, Tse Sum Bui B, Wolff M, Miginiac-Maslow M, Rohmer M (2006) Isoprenoid biosynthesis in plant chloroplasts via the MEP pathway: direct 
thylakoid/ferredoxin-dependent photoreduction of GcpE/lspG. FEBS Lett 580:1547-1552. doi:10.1016/j.febslet.2006.01.082

Sharma SK, Le Maguer M (1996) Kinetics of lycopene degradation in tomato pulp solids under different processing and storage conditions. Food Res Int 29:309-315. doi:10.1016/0963-9969(96)00029-4

Wang GS, Grammel H, Abou-Aisha K, Sägesser R, Ghosh R (2012) High-level production of the industrial product Lycopene by the photosynthetic Bacterium Rhodospirillum rubrum. Appl Environ Microbiol 78:7205-7215. doi:10.1128/AEM.00545-12

Xu F, Yuan QP, Zhu Y (2007) Improved production of lycopene and b-carotene by Blakeslea trispora with oxygen-vectors. Process Biochem 42:289-293. doi:10.1016/j.procbio.2006.08.007

Yoon K-W, Doo E-H, Kim S-W, Park J-B (2008) In situ recovery of lycopene during biosynthesis with recombinant Escherichia coli. J Biotechnol 135:291-294. doi:10.1016/j.jbiotec.2008.04.001

Yoon SH, Lee SH, Das A, Ryu HK, Jang HJ, Kim JY, Oh DK, Keasling JD, Kim SW (2009) Combinatorial expression of bacterial whole mevalonate pathway for the production of $\beta$-carotene in E. coli. J Biotechnol 140:218-226. doi:10.1016/j.jbiotec.2009.01.008

Yoon SH, Han M-J, Jeong H, Lee CH, Xia X-X, Lee D-H, Shim JH, Lee SY, Oh TK, Kim JF (2012) Comparative multi-omics systems analysis of Escherichia coli strains B and K-12. Genome Biol 13:R37. doi:10.1186/ gb-2012-13-5-r37

Yuan LZ, Rouvière PE, LaRossa RA, Suh W (2006) Chromosomal promoter replacement of the isoprenoid pathway for enhancing carotenoid production in E. coli. Metab Eng 8:79-90. doi:10.1016/j.ymben.2005.08.005

Zhang C, Chen X, Zou R, Zhou K, Stephanopoulos G, Too HP (2013) Combining genotype improvement and statistical media optimization for isoprenoid production in E. coli. PLoS One. doi:10.1371/journal.pone.0075164

Zhou K, Zou R, Stephanopoulos G, Too HP (2012) Metabolite profiling identified methylerythritol cyclodiphosphate efflux as a limiting step in microbial isoprenoid production. PLoS One. doi:10.1371/journal.pone.0047513

\section{Submit your manuscript to a SpringerOpen ${ }^{\odot}$ journal and benefit from:}

- Convenient online submission

$\checkmark$ Rigorous peer review

- Immediate publication on acceptance

- Open access: articles freely available online

- High visibility within the field

- Retaining the copyright to your article

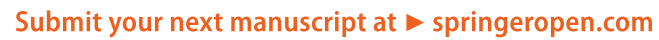

